Proceeding Series of the Brazilian Society of Computational and Applied Mathematics

\title{
Software para Simulação de Crescimento de Microalgas em Fotobiorreatores Tubulares
}

\author{
Robert Luis Lara Ribeiro
}

Universidade do Estado do Amazonas, UEA, Tefé, AM

André Bellin Mariano ${ }^{2}$

Núcleo de Pesquisa e Desenvolvimento de Energia Auto-Sustentável, UFPR, Curitiba, PR

José Viriato Coelho Vargas ${ }^{3}$

Departamento de Engenharia Mecânica, UFPR, Curitiba, PR

\begin{abstract}
Resumo. Microalgas são apresentadas como fonte de energia alterativa ao petróleo, sendo capaz de transformar a energia solar em biodiesel. Desta forma, o Núcleo de Pesquisa e Desenvolvimento em Energia Auto-Sustentável (NPDEAS/UFPR) está construindo fotobiorreatores tubulares para cultivar estes microorganismos, também elabora um software para simular o crescimento microalgal.
\end{abstract}

Palavras-chave. Software, Simulação Computacional, Modelo Matemático, Fotobiorreatores, Crescimento de Microalgas.

\section{Introdução}

As microalgas são apresentadas como fonte de energia alternativa ao petróleo, com capacidade de produção de energia através da forma de biocombustível. Assim diversas Universidades e Institutos de pesquisa estão investindo cada vez mais tempo de pesquisa e recursos para que os pesquisadores possam extrair o máximo de biodiesel possível a partir destes microorganismos. Em comparação com a produção de sementes oleaginosas que diz respeito à produção de biodiesel, algumas culturas de microalgas têm inúmeras vantagens devido a sua biodiversidade, tais como, podem dobrar sua biomassa em pequeno espaço de tempo, em fotobiorreatores bem estruturados podem absorver grande quantidade de energia solar, têm lipídeos de alta densidade, fornecem uma produtividade (litros de óleo produzido por hectare por ano) de até dez vezes maior do que as oleaginosas podendo ser cultivadas em áreas não apropriadas para a agricultura e geram menos resíduos $[2,5,10]$.

\footnotetext{
${ }^{1}$ robertlarabr@gmail.com

2 andrebmariano@gmail.com

3 vargasjvcv@gmail.com
} 
Fatores como radiação solar incidente nos tubos, $\mathrm{pH}$, nutrientes e temperatura do meio de cultivo comprometem fortemente o cultivo das microalgas, pois influenciam diretamente na composição celular do microorganismo. Ter um fotobiorreator que seja capaz de controlar estes fatores é de suma importância para obter maiores rendimentos de biomassa microalgal $[3,7,9]$. O pH e a composição de nutrientes no meio de cultivo pode ser controlado facilmente por dispositivos instalados no fotobiorreator, no entanto, a radiação solar incidente e a temperatura no meio de cultivo são mais difíceis de controlar, quando trabalhamos com fotobiorreatores construídos ao ar livre, pois estes fatores dependem de condições ambientais diversas como velocidade dos ventos, ângulo solar, umidade do ar, temperatura local, etc. $[1,4,6]$.

Estre trabalho busca apresentar um software capaz de simular computacionalmente a dinâmica do crescimento de microalgas em fotobiorreatores tubulares construídos no NPDEAS.

\section{Materiais e Métodos}

\subsection{Fotobiorreatores tubulares construídos no NPDEAS}

O NPDEAS, localizado na Universidade Federal do Paraná, está construindo FBRT para cultivar microalgas, com o objetivo de obter biodiesel a partir da estrutura lipídica destes microorganismos [8].

A estrutura dos FBRT é composta de $3.710 \mathrm{~m}$ de tubos transparentes de PVC cristal, estes tubos estão distribuídos em uma geometria compacta arranjada em uma matriz de 14 colunas por 53 linhas de tubos (total de 742 tubos), onde cada tubo tem $5 \mathrm{~m}$ de comprimento, com um raio de 0,03 m, conforme Figura 1.a. Este fotobiorreator é considerado compacto por ter uma capacidade de aproximadamente 12.600 litros de meio de cultivo microalgal, usando apenas $10 \mathrm{~m}^{2}$ de área construída. Outra característica importante destes fotobiorreatores é ter uma área lateral de $122 \mathrm{~m}^{2}$ de exposição à radiação solar incidente.

a)

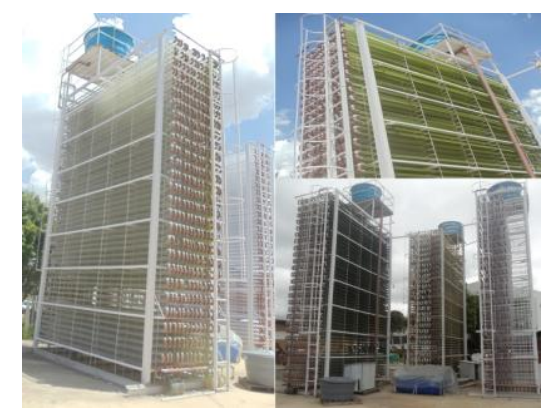

b)

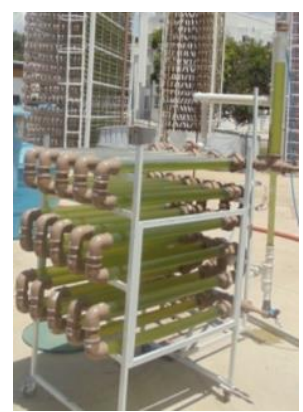

Figura 1: a) FBRT do NPDEAS. b) Protótipo do FBRT do NPDEAS.

Foi construído também um FBRT em escala menor, ou seja, um protótipo do fotobiorreator tubular grande. Este fotobiorreator menor tem a vantagem de ser mais estável que os fotobiorreatores maiores, no entanto, por ser menor têm produtividades menores em relação à área utilizada para a sua construção. Sua estrutura é composta por 30 tubos de $1 \mathrm{~m}$ de comprimento, sendo distribuídos em 5 colunas e 6 linhas. Sua capacidade é de 105 litros 
de meio de cultivo microalgal, tendo como área de construção $1,5 \mathrm{~m}^{2}$, conforme Figura 1.b.

Além da estrutura em si, os FBRT são compostos por bombas hidráulicas que são responsáveis pela circulação do meio de cultivo microalgal em seu interior, e por um sistema de aeração que fornece ar atmosférico enviado por compressores.

\subsection{Microalga}

A microalga utilizada pela equipe do NPDEAS é uma espécie do gênero Scenedesmus que foi isolada da rede de abastecimento de água de Curitiba e, portanto, apresenta como vantagem a pré-adaptação às condições de cultivo em ambiente externo, aliada ao baixo risco na geração de impacto ambiental em caso de vazamento, uma vez que se trata de uma espécie natural da região.

Nos experimentos realizados nos FBR necessitou-se de uma pré-cultura de microalgas para servir de inoculo para os experimentos principais. A produção do inoculo consiste de duas etapas preliminares, na primeira realizou-se o crescimento da cultura em frascos Erlenmeyer de $2 \mathrm{~L}$, logo após, na segunda etapa o cultivo é transferido para galões de $20 \mathrm{~L}$ para em seguida realizar a inoculação nos FBRs.

Em todas as etapas de cultivo utilizou-se o meio CHU de nutrientes, que é composto pelos macro e micronutrientes necessários para o crescimento das microalgas.

\subsection{Modelo matemático}

Para estimar a produção de biodiesel de um FBRT é necessário inicialmente calcular a concentração de biomassa microalgal. Desta forma, divide-se o FBRT em cinco diferentes tipos de componentes físicos, conforme a Figura 2:

Tipo 1 - Tubos transparentes

Tipo 2 - Reservatório

Tipo 3 - Bomba

Tipo 4 - Tubos opacos

Tipo 5 - Coluna de Gaseificação

Em cada um dos cinco tipos de componentes físicos irá ocorrer crescimento microalgal, no entanto, em alguns podem existir limitações no crescimento. Assim, cada componente físico é subdividido em Elementos de Volume (EV), sendo que o domínio a ser analisado é transformado em células de volume centradas que estabelece uma única equação diferencial ordinária para cada célula através da Lei de Conservação de Espécies entre os EV.

Todos os componentes físicos são divididos em dois EV (um para a parede dos componentes físicos o qual é chamado de $\mathrm{EV}_{\mathrm{w}}$ e outro para a parte interna do tubo onde etrará fluido que é chamado de $\mathrm{EV}_{\mathrm{f}}$ ). 


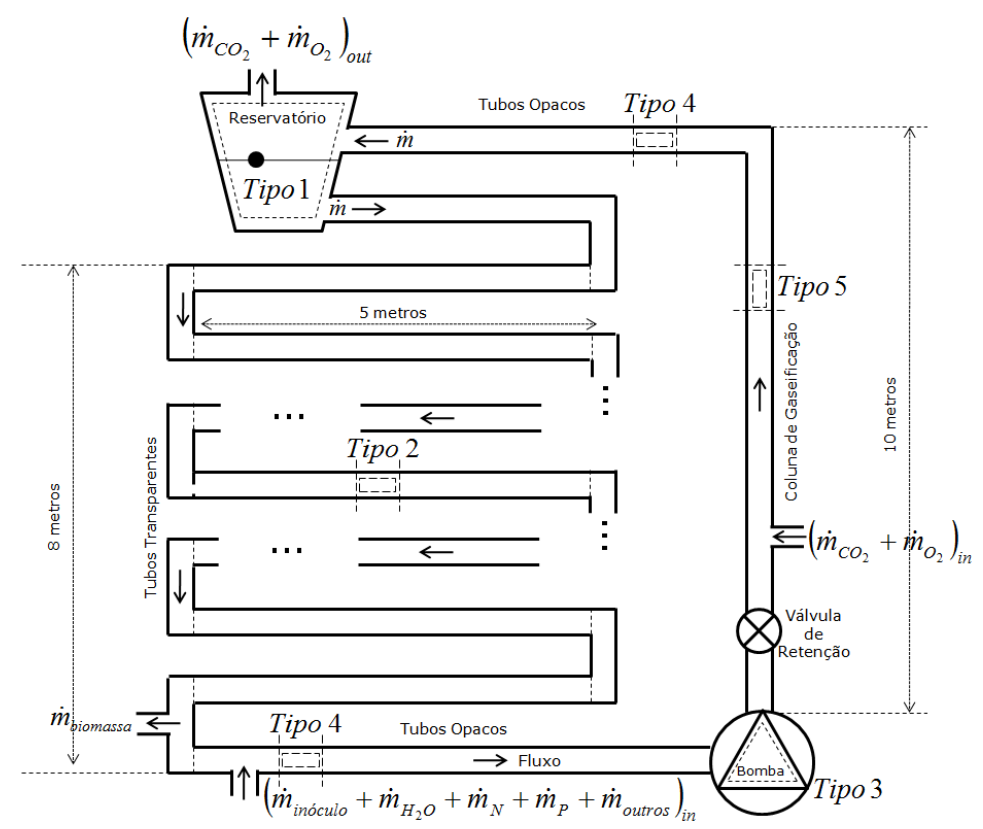

Figura 2: Sistema físico simplificado do fotobiorreator.

Desta forma as Equações Diferenciais Ordinárias que formam o modelam matematicamente o sistema de cultivos de microalgas em Fotobiorreator Tubular é:

$$
\left\{\begin{array}{l}
\frac{d Y_{1}^{(i, j)}}{d t}=\frac{\dot{m}}{V_{f}^{(i, j)} \rho_{f}}\left(Y_{1}^{(i, j-1)}-Y_{1}^{(i, j)}\right)+Y_{1, \text { ger }}^{(i, j)}-c_{1, \text { out }} Y_{1, \text { out }} \\
\frac{d Y_{2}^{(i, j)}}{d t}=\frac{\dot{m}}{V_{f}^{(i, j)} \rho_{f}}\left(Y_{2}^{(i, j-1)}-Y_{2}^{(i, j)}\right)-Y_{2, \text { con }}^{(i, j)}+c_{2, i n} Y_{2, \text { in }} \\
\frac{d Y_{3}^{(i, j)}}{d t}=\frac{\dot{m}}{V_{f}^{(i, j)} \rho_{f}}\left(Y_{3}^{(i, j-1)}-Y_{3}^{(i, j)}\right)+Y_{3, g e r}^{(i, j)}+c_{3, \text { in }} Y_{3, \text { in }} \\
\frac{d Y_{4}^{(i, j)}}{d t}=\frac{\dot{m}}{V_{f}^{(i, j)} \rho_{f}}\left(Y_{4}^{(i, j-1)}-Y_{4}^{(i, j)}\right)-Y_{4, \text { con }}^{(i, j)}+c_{4, i n} Y_{4, \text { in }} \\
\frac{d T_{w}^{(i, j)}}{d t}=\frac{k_{w} A_{s, w}}{m_{w}^{(j)} c_{w}}\left(T_{w}^{(i, j-1)}-T_{w}^{(i, j)}\right)+\frac{-\dot{Q}^{(i, j)}-\dot{Q}_{a r}^{(i, j)}+\dot{Q}_{r a d}^{(i, j)}}{m_{w}^{(i, j)} c_{w}} \\
\frac{d T_{f}^{(i, j)}}{d t}=\frac{\dot{m}}{m_{f}^{(i, j)}}\left(T_{f}^{(i, j-1)}-T_{f}^{(i, j)}\right)+\frac{\dot{Q}_{r a d, \tau}^{(i, j)}-\dot{Q}_{r a d, a l g}^{(i, j)}+\dot{Q}^{(i, j)}}{m_{f}^{(i, j)} c_{f}}
\end{array}\right.
$$

sendo $i=1,2,3,4$ ou 5 representando o Tipo de EV e $j=1,2, \ldots, n$ representando o número do EV.

O modelo matemático apresentado no sistema de equações diferenciais utiliza os princípios físicos presentes em Dinâmica Populacional, Cinética Química, Termodinâmica clássica e correlações empíricas de Mecânica dos Fluídos, Transferência de Calor e Massa e também Óptica. 


\subsection{Software para simulação}

Foi desenvolvida uma interface gráfica para o software de simulação de cultivo de microalgas em fotobiorreatores tubulares compactos. O código da interface foi escrito em linguagem Java, e tem como finalidade facilitar o uso acadêmico e industrial.

Na Figura 3 apresenta-se a tela no simulador onde é possível escolher algumas opções quanto a visualização da simulação em 3D.

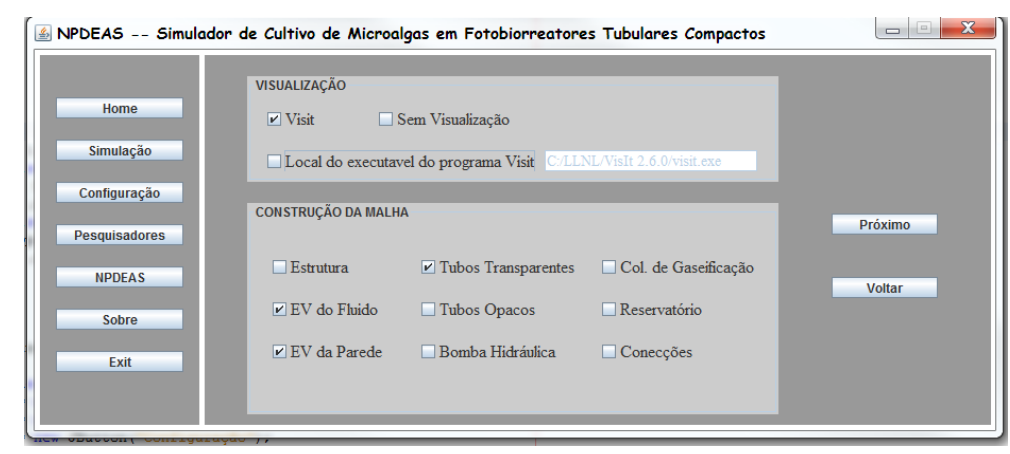

Figura 3: Configurações de visualização da malha.

O usuário pode entrar com os detalhes sobre da geometria do FBR, conforme a tela da Figura 4, podendo escolher o número de linhas, colunas, pontos para formar a circunferência da parede do tubo, número de EV por tubo, distância entre os tubos, comprimento do tubo, raio interno e externo e a posição espacial do primeiro tubo.

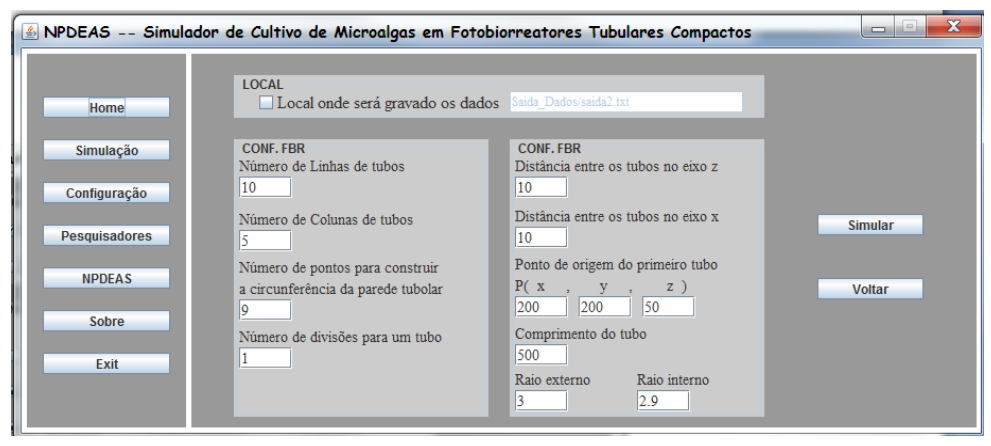

Figura 4: Configurações da malha dos tubos.

Esta interface gráfica simples serve para ajudar a usuários leitos em programação a realizar simulações de cultivo de microalgas nos FBR Tubulares Compactos com maior facilidade.

\section{Resultados e Discussão}

Realizou-se uma simulação computacional utilizando as mesmas condições iniciais, geométricas e operacionais de um experimento realizado durante 24 dias no Fotobiorreator 
Tubular. O cultivo inicia-se com 4 colunas em atividade e após 16 dias são adicionada outras 4 colunas, acarretando em uma diluição da biomassa em $50 \%$ de sua concentração. O gráfico dos resultados numéricos com os resultados experimentais é mostrado na Figura 5.

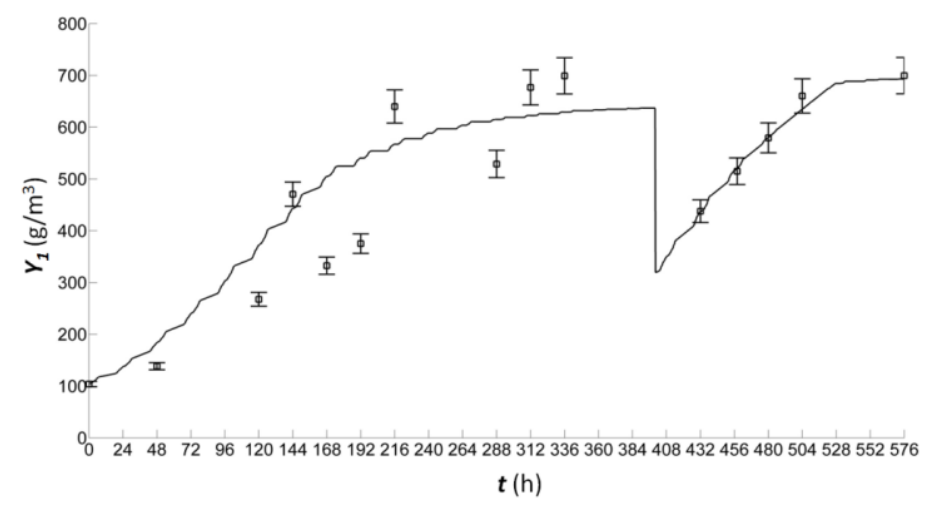

Figura 5: Concentração numérica e experimental no fotobiorreator com $\mathrm{r}^{2}=0,82969$.

A Figura 6 apresenta quatro resultados numéricos de concentração de microalgas, o primeiro resultado é o início da simulação com concentração inicial de $104 \mathrm{~g} / \mathrm{m}^{3}$, o segundo resultado é referente a 380 horas de simulação, sua concentração é de $635,8 \mathrm{~g} / \mathrm{m}^{3}$. O terceiro resultado apresenta o momento que a quinta, sexta, sétima e oitava colunas de tubos são abertas, desta forma, toda a concentração de microalgas é diluída. O quarto resultado numérico é o final da simulação e obtém concentração igual a $693,4 \mathrm{~g} / \mathrm{m}^{3}$.
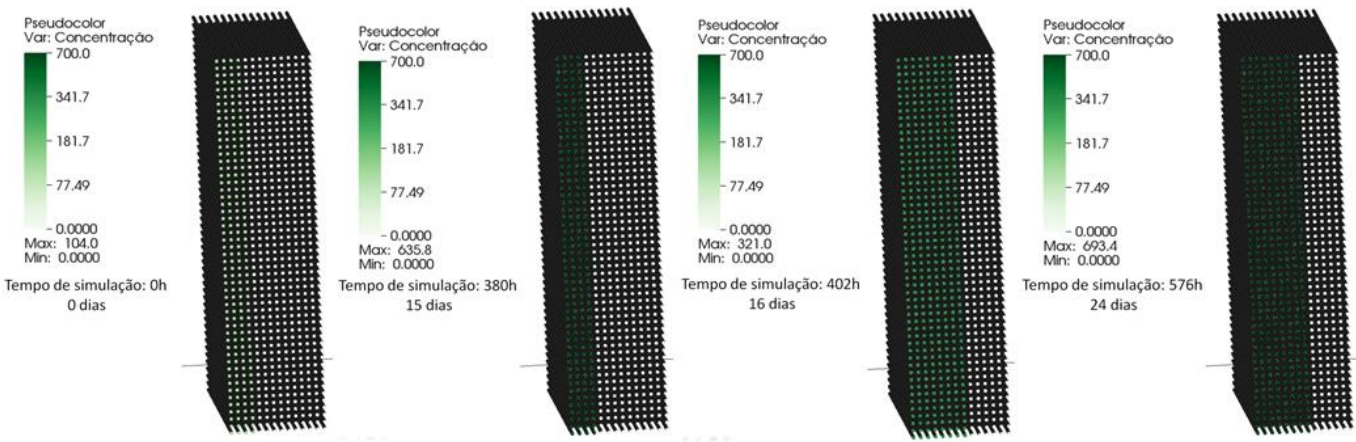

Figura 6: Quatro resultados de concentração de microalgas pela simulação numérica.

\section{Conclusão}

Pode-se concluir que o modelo matemático e computacional apresenta resultados de perfis de concentrações qualitativamente satisfatórios para cultivos realizados nos FBR tubular. O software em desenvolvimento auxilia de forma significativa, acelerando o processo de simulação e construção das malhas dos FBR tubulares. 


\section{Agradecimentos}

Os autores agradecem o apoio da equipe do NPDEAS, CNPq, NILKO e UFPR.

\section{Referências}

[1] M. Bereguel, F. Rodriguez and J. L. Garcia, Model predictive control of $\mathrm{pH}$ in tubular potobioreactors, Journal of Process Control, vol. 14, 377-387, (2014).

[2] Y. Chisti, Biodiesel from microalgae, Biotechnology Advances, vol. 25, 294-306, (2007).

[3] Y. Kitaya, H. Azuma and M. Kiyota, Effects of temperature, $\mathrm{CO} 2 / \mathrm{O} 2$ concentrations and light intensity on cellular multiplication of microalgae, Euglena gracilis, Advances in Space Research, vol. 35, 1584-1588, (2005).

[4] A. Kunjapur and R. Eldridge, Photobioreactor design for commercial biofuel production from microalgae, Industrial \& Engineering Chemistry Research, vol. 49, 3516-3526, (2010).

[5] S. O. Lourenço, Cultivo de microalgas marinhas - princípios e aplicações, Editora RiMa, (2006).

[6] M. Morweiser, C. Posten and O. Kruse, Developments and perspectives of photobioreactors for biofuel production, Applied Microbiology and Biotechnology, vol. 87, 1291-1301, (2010).

[7] J. F. Sánchez, E. M. Grima, F. G. Acién, J. M. F. Sevilla, J. P. Parra and M. C. Cerón, Biomass and lutein productivity of Scenedesmus almeriensis: influence of irradiance, dilution rate and temperature, Applied Microbiology and Biotechnology, vol. 79, 719729, (2008).

[8] K. G. Satyanarayana1, A. B. Mariano and J. V. C. Vargas, A review on microalgae, a versatile source for sustainable energy and materials, International Journal of Energy Research, vol. 35, 291-311, (2011).

[9] C. Ugwu, H. Aoyagi and H. Uchiyama, Photobioreactors for mass cultivation of algae, Bioresource Technology, vol. 99, 4021-4028, (2008).

[10] H. Xu, Q. Wu and X. Miao, High quality biodiesel production from a microalga Chlorella protothecoides by heterotrophic growth in fermenters, Journal of Biotechnology, vol. 126, 499-507, (2006). 